\title{
Neutral Hydrogen in the Bar of NGC 4151
}

\author{
C. G. Mundell, A. Pedlar and D. L. Shone \\ University of Manchester, Nuffield Radio Astronomy Laboratories, \\ Jodrell Bank, Macclesfield, Cheshire SK11 9DL, U.K.
}

M. W. Asif

Royal Greenwich Observatory, Madingly Rd., Cambridge CB3 OEZ, UK

A. Robinson

University of Cambridge, Institute of Astronomy, Madingly Road, Cambridge CB3 OHA, U.K.

D. J. Axon

Space Telescope Science Institute, 3700 San Martin Dr., Baltimore, MD 21218

\section{Introduction}

Bars are thought to play a crucial role in the fueling of AGN (see e.g., Mundell et al., in these proceedings and references therein), and as part of a project to investigate this, we have studied the neutral hydrogen structure in the bar of the Seyfert 1.5 galaxy NGC 4151.

\section{Observations and Discussion}

High-sensitivity VLA observations have enabled us to image the neutral hydrogen emission from the bar of NGC 4151 in unprecedented detail, and is a continuation of the work by Pedlar et al. (1992).

Figure 1 shows images of integrated neutral hydrogen emission. The large scale emission extends over approximately $20 \mathrm{kpc}$ and the outer part consists of a well-defined two-armed spiral which originates from a fat bar at a radius of about $5 \mathrm{kpc}$. The bar shows complex structurc, including two sharp features which are reminiscent of the shocks seen in many simulations, e.g., Athanassoula (1992). These features appear to join the central 1-kpc ring discovered by VilaVilaró et al. (1994), and for which there appears a partial counterpart in neutral hydrogen emission.

Recent MERLIN observations of neutral hydrogen absorption (Mundell et al. 1995) have revealed evidence for a torus of neutral hydrogen, $50 \mathrm{pc}$ in size which is thought to be a part of the fuel-chain leading to the active nucleus, and also responsible for collimating the nuclear $U V$ radiation. Work is in progress to follow the neutral hydrogen emission structure to the region within the 1-kpc ring and hence infer its relation, if any, to the the proposed torus. In addition, 


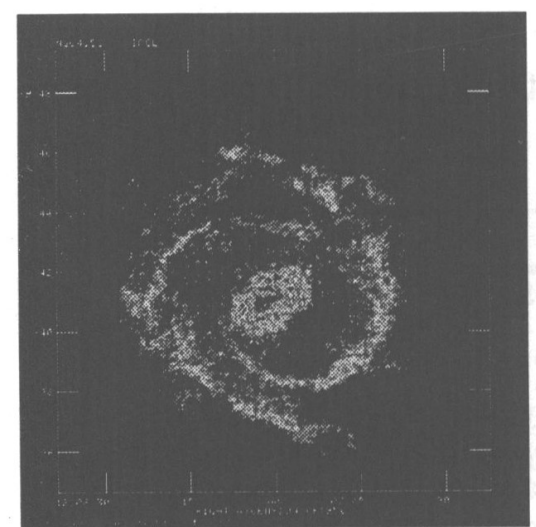

AIPS Usor 832

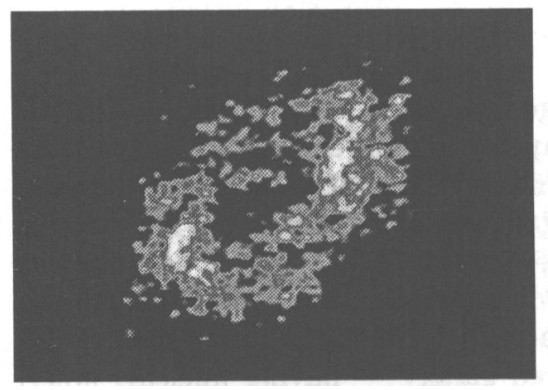

High-resolution image of the bar in NGC4151 showing bright shock-like features.

Figure 1. VLA Images of the NGC 4151

we are studying the detailed spectral structure of the bar to look for signs of streaming motions.

\section{References}

Athanassoula, E. 1992, MNRAS, 259, 345

Mundell, C. G., Pedlar, A., Baum, S. A., O'Dea, C. P., Gallimore, J. F., \& Brinks, E. 1995 MNRAS, 275, 67

Pedlar, A., Howley, P., Axon., D. J. \& Unger, S. W. 1992 MNRAS, 259, 369P Vila-Vilaró, B., Robinson, A., Perez, E., Axon, D. J., Baum, S. A., G onzálezDelgado, R. M., Pedlar, A. et al. 1994, A\&A, 302, 58V 